\title{
Beam-ACO Based on Stochastic Sampling: A Case Study on the TSP with Time Windows
}

\author{
Manuel López-Ibáñez and Christian Blum \\ ALBCOM, Dept. Llenguatges i Sistemes Informàtics, Universitat Politècnica de \\ Catalunya, Barcelona, Spain. E-mail: \{m.lopez-ibanez, cblum\}@lsi.upc.edu
}

\begin{abstract}
Beam-ACO algorithms are hybrid methods that combine the metaheuristic ant colony optimization with beam search. They heavily rely on accurate and computationally inexpensive bounding information for choosing between different partial solutions during the solution construction process. In this work we present the use of stochastic sampling as a useful alternative to bounding information in cases were computing accurate bounding information is too expensive. As a case study we choose the well-known travelling salesman problem with time windows. Our results clearly demonstrate that Beam-ACO, even when bounding information is replaced by stochastic sampling, may have important advantages over standard ACO algorithms.
\end{abstract}

\section{Introduction}

Ant colony optimization (ACO) is a metaheuristic that is based on the probabilistic construction of solutions [1]. At each algorithm iteration, $n$ solutions are constructed independently from each other. A recently proposed ACO hybrid, known as Beam-ACO [2,3], employs at each iteration a probabilistic beam search procedure that constructs $n$ solutions non-independently in parallel. A crucial component of beam search is bounding information for choosing between different partial solutions at each step of the solution construction process [4]. At each step, beam search keeps a certain number of the best partial solutions available for further extension, and excludes the rest from further examination. A problem arises when bounding information is either misleading (that is, the wrong partial solutions are kept for further examination) or when bounding information is computationally expensive.

Browsing the relevant artificial intelligence literature, we came across a different method for the evaluation of partial solutions in the context of tree search procedures: probing or stochastic sampling [5,6]. Hereby, each given partial solution is completed a number of $N^{\mathrm{s}}$ times in a stochastic way. The information that is obtained in this way is used to differentiate between different partial solutions.

In this work we propose to replace the use of bounding information in Beam$\mathrm{ACO}$ with a stochastic sampling procedure. For this case study we choose the travelling salesman problem with time windows (TSPTW), due to the fact that 
accurate bounding information that is computationally inexpensive is - to our knowledge - not available for this problem. Finally, we want to state clearly at this point that the primary goal of this research is not to obtain state-of-the-art results for the TSPTW, which is left for future work. Our aim is to show that Beam-ACO based on stochastic sampling may have significant advantages over standard ACO algorithms.

The remainder of this work is organized as follows. In Section 2 we give a technical description of the TSPTW. Furthermore, in Sections 3 and 4 we first introduce our standard (in the sense of non-hybrid) ACO algorithm for the TSPTW and then we introduce the probabilistic beam search procedure that is needed for Beam-ACO. Finally, in Section 5 we present an experimental evaluation, and in Section 6 we offer conclusions and an outlook to future work.

\section{The TSPTW}

The traveling salesman problem with time windows (TSPTW) is the problem of finding an efficient route to visit a number of customers, starting and ending at a depot, with the added difficulty that each customer may only be visited within a certain time window. In practice, the TSPTW is an important problem in logistics.

The TSPTW is proven to be NP-hard, and even finding a feasible solution is an NP-complete problem [7]. The problem is closely related to a number of important problems. For example, the well-known traveling salesman problem (TSP) is a special case of the TSPTW. The TSPTW itself can be seen as a special case with a single vehicle of the vehicle routing problem with time windows (VRPTW). The state of the art in solving the TSPTW is a simulated annealing approach by Ohlmann and Thomas [8].

\subsection{Formal Problem Definition}

The TSPTW is formally defined as follows. Let $G=(N, A)$ be a finite graph, where $N=\{0,1, \ldots, n\}$ consists of a set of nodes representing the depot (node 0 ) and $n$ customers, and $A=N \times N$ is the set of arcs connecting the nodes.

For every arc $a_{i j} \in A$ between two nodes $i$ and $j$, there is an associated cost $c\left(a_{i j}\right)$. This cost typically represents the travel time between customers $i$ and $j$, plus a service time at customer $j$.

For every node $i \in N$, there is an associated time window, $\left[e_{i}, l_{i}\right]$, where $e_{i}$ represents the earliest service start time and $l_{i}$ is the latest service start time.

A solution to the problem is a tour visiting each node once, starting and ending at the depot. Hence, a tour is represented as $P=\left(p_{0}=0, p_{1}, \ldots, p_{n}, p_{n+1}=\right.$ $0)$, where the sub-sequence $\left(p_{1}, \ldots, p_{k}, \ldots, p_{n}\right)$ is a permutation of the nodes in $N \backslash\{0\}$ and $p_{k}$ denotes the index of the customer at the $k^{\text {th }}$ position of the tour. Two additional elements, $p_{0}=0$ and $p_{n+1}=0$, represent the starting and final depot. 
It is assumed that waiting times are permitted, that is, a node $i$ can be reached before the start of its time window $e_{i}$, but cannot be left before $e_{i}$. Therefore, the departure time from customer $p_{k}$ is calculated as $D_{p_{k}}=\max \left(A_{p_{k}}, e_{p_{k}}\right)$, where $A_{p_{k}}=D_{p_{k-1}}+c\left(a_{p_{k-1}, p_{k}}\right)$ is the arrival time at the customer $p_{k}$ in the tour.

The literature defines two related but different objectives for this problem. One is the minimization of the cost of the arcs traversed along the tour $\sum_{k=0}^{n} c\left(a_{p_{k}, p_{k+1}}\right)$. The other alternative is to minimise $A_{p_{n+1}}$, the arrival time at the depot. In this work, we focus on the former, and, therefore, we formally defined the TSPTW as:

$$
\text { minimise: } \quad F(P)=\sum_{k=0}^{n} c\left(a_{p_{k}, p_{k+1}}\right)
$$

subject to:

$$
\Omega(P)=\sum_{k=0}^{n+1} \omega\left(p_{k}\right)=0
$$

where:

$$
\begin{aligned}
& \omega\left(p_{k}\right)= \begin{cases}1 & \text { if } A_{p_{k}}>l_{p_{k}}, \\
0 & \text { otherwise; }\end{cases} \\
& A_{p_{k+1}}=\max \left(A_{p_{k}}, e_{p_{k}}\right)+c\left(a_{p_{k}, p_{k+1}}\right) .
\end{aligned}
$$

In the above definition, $\Omega(P)$ denotes the number of time window constraints that are violated by tour $P$, which must be zero for feasible solutions.

\section{The ACO Algorithm}

The application of the ACO framework to any problem implies the definition of a solution construction mechanism and the specification of appropriate pheromone information $\mathcal{T}$.

In the case of the TSPTW, ants construct a complete tour by starting at the depot (node 0) and iteratively adding customers to the tour. Once all customers have been added to the tour, it is completed by adding node 0 .

As for the pheromone information, $\forall a_{i j} \in A, \exists \tau_{i j} \in \mathcal{T}, 0 \leq \tau_{i j} \leq 1$, where $\tau_{i j}$ represents the desirability of visiting customer $j$ after customer $i$ in the tour: the greater the pheromone value $\tau_{i j}$, the greater is the desirability of choosing $j$ as the next customer to visit in the current tour.

The particular ACO algorithm proposed in this paper for the TSPTW combines ideas from both $\mathcal{M M A S}$ and ACS algorithms implemented in the hypercube framework (HCF) as proposed by Blum and Dorigo [9]. A high level description of the algorithm is given in Algorithm 1. The data structures used, in addition to counters and to the pheromone values, are: (1) the best-so-far solution $P^{\mathrm{bf}}$, i.e., the best solution generated since the start of the algorithm; (2) the restart-best solution $P^{\mathrm{rb}}$, that is, the best solution generated since the 


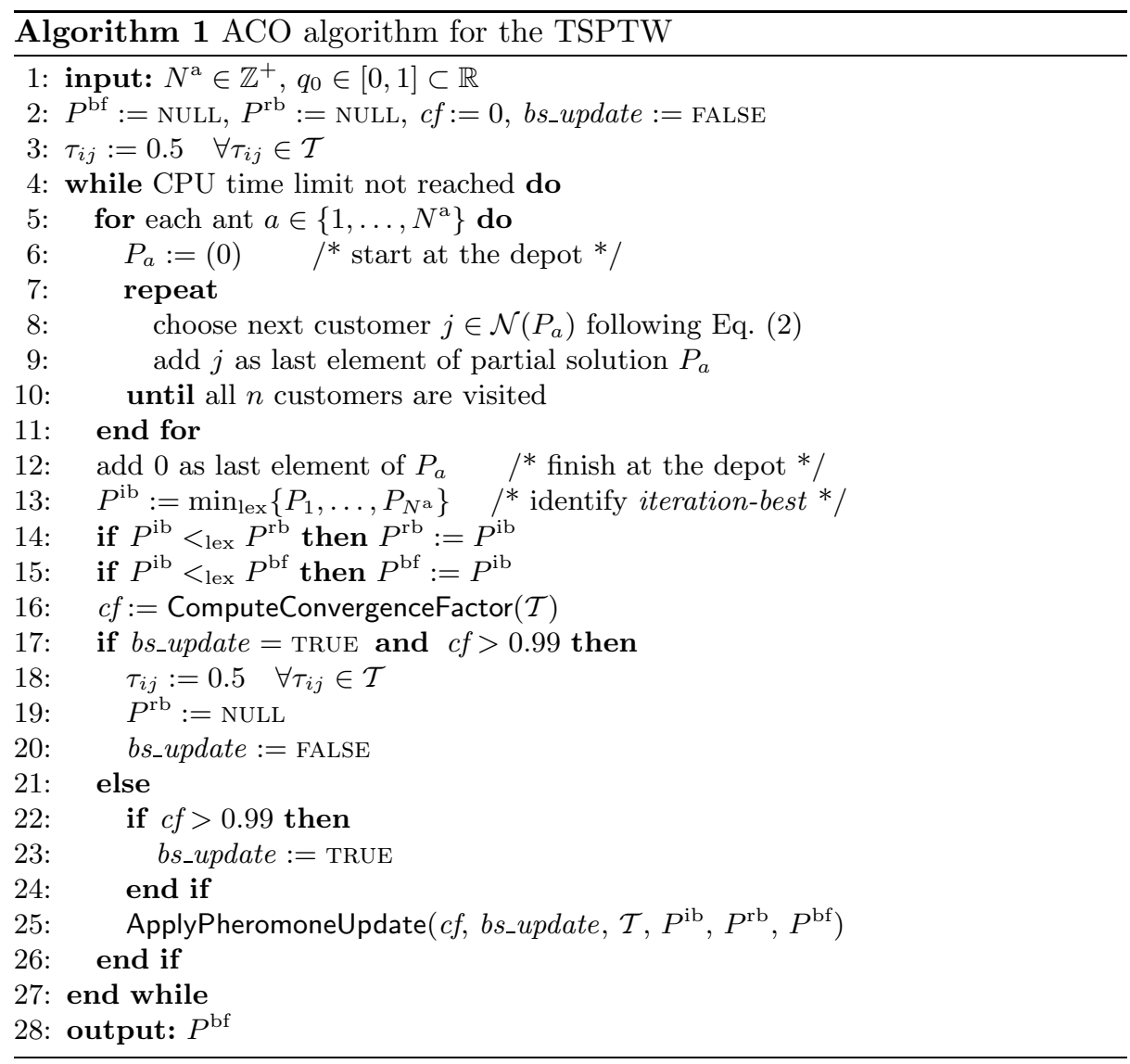

last restart of the algorithm; (3) the convergence factor $(c f), 0 \leq c f \leq 1$, which is a measure of how far the algorithm is from convergence; and (4) the Boolean variable bs_update, which becomes true when the algorithm reaches convergence.

Roughly, the algorithm works as follows. Initially, all variables are initialized. In particular, the pheromone values are set to their initial value 0.5 . Then, a main loop is repeated until a termination criteria, such as a CPU time limit, is met. Each algorithm iteration consists of the following steps.

First, a number of ants $\left(N^{\mathrm{a}}\right)$ construct complete tours by following the state transition rule defined in Eq. (2). Each ant $a$ constructs a single tour $P_{a}$ by iteratively adding customers to its partial tour. At each construction step, ant $a$ chooses one customer $j$ among the set $\mathcal{N}\left(P_{a}\right)$ of customers not visited yet by the current partial tour $P_{a}$. The decision is made by firstly generating a random number $q$ uniformly distributed within $[0,1]$ and comparing this value with a parameter $q_{0}$ called the determinism rate. If $q \leq q_{0}, j$ is chosen deterministically as the value with the highest product of pheromone and heuristic information. Otherwise, $j$ is stochastically chosen from a distribution of probabilities. This 
rule is described by the following equation:

$$
\begin{cases}j=\arg \max _{k \in \mathcal{N}\left(P_{a}\right)}\left\{\tau_{i k} \cdot \eta_{i k}\right\} & \text { if } q \leq q_{0}, \\ j \sim\left\{\mathbf{p}_{i}(k) \mid k \in \mathcal{N}\left(P_{a}\right)\right\} & \text { otherwise. }\end{cases}
$$

where $i$ is the last customer added to the tour $P_{a}, \eta_{i j}$ is a heuristic value that represents an estimation of the benefit of visiting customer $j$ after customer $i$, and the symbol $\sim$ denotes drawing a random number from a probability distribution defined by the probabilities $\mathbf{p}_{i}(k)$. These probabilities depend on the pheromone and heuristic information associated to each choice and are defined by the following probabilistic rule:

$$
\mathbf{p}_{i}(j)=\frac{\tau_{i j} \cdot \eta_{i j}}{\sum_{k \in \mathcal{N}\left(P_{a}\right)} \tau_{i k} \cdot \eta_{i k}} \quad \text { if } j \in \mathcal{N}\left(P_{a}\right)
$$

For the TSPTW, we define a heuristic information that combines the travel cost between customers $\left(c_{i j}\right)$ and the latest service time $\left(l_{j}\right)$. The values are first normalized to $[0,1]$, with the maximum value corresponding to 0 and the minimum to 1 , and then combined with equal weight:

$$
\eta_{i j}=\frac{1}{2}\left(\frac{c^{\max }-c_{i j}}{c^{\max }-c^{\min }}+\frac{l^{\max }-l_{j}}{l^{\max }-l^{\min }}\right)
$$

After all ants have completed their tours, the tours are compared to identify the iteration-best solution $\left(P^{\mathrm{ib}}\right)$, i.e., the best solution among the ones constructed in the current iteration, denoted as $\min _{\text {lex }}=\left\{P_{1}, \ldots, P_{N^{a}}\right\}$. To identify the best solution, tours are compared lexicographically $\left(<_{l e x}\right)$ by first minimising the number of constraint violations $(\Omega)$ and, if they have equal number of constraint violations, comparing their tour cost $(F)$. More formally, we compare two different tours $P$ and $P^{\prime}$ as follows:

$$
P<_{\operatorname{lex}} P^{\prime} \Longleftrightarrow \Omega(P)<\Omega\left(P^{\prime}\right) \vee\left(\Omega(P)=\Omega\left(P^{\prime}\right) \wedge F(P)<F\left(P^{\prime}\right)\right)
$$

Next, a new value for the convergence factor $c f$ is computed. Depending on this value, as well as on the value of the Boolean variable bs_update, a decision on whether to restart the algorithm or not is made. If the algorithm is restarted, all the pheromone values are reset to their initial value (0.5). The algorithm is iterated until the CPU time limit is reached. Once terminated, the algorithm returns the best solution found which corresponds to $P^{\mathrm{bf}}$. In the following we describe the two remaining procedures of Algorithm 1 in more detail.

Procedure ComputeConvergenceFactor $(\mathcal{T})$ computes the convergence factor $c f$, which is a function of the current pheromone values, as follows:

$$
c f=2\left(\frac{\sum_{\tau_{i j} \in \mathcal{T}} \max \left\{\tau^{\max }-\tau_{i j}, \tau_{i j}-\tau^{\min }\right\}}{|\mathcal{T}| \cdot\left(\tau^{\max }-\tau^{\mathrm{min}}\right)}-0.5\right)
$$


Table 1. Setting of $\kappa^{\text {ib }}, \kappa^{\text {rb }}$ and $\kappa^{\text {bf }}$ depending on the convergence factor $c f$ and the Boolean control variable bs_update.

\begin{tabular}{c|cccc|c}
\hline $\begin{array}{c}\text { bs_update } \\
c f\end{array}$ & {$[0,0.4)$} & {$[0.4,0.6)$} & {$[0.6,0.8)$} & {$[0.8,1]$} & - \\
\hline$\kappa^{\mathrm{ib}}$ & 1 & $2 / 3$ & $1 / 3$ & 0 & 0 \\
$\kappa^{\mathrm{rb}}$ & 0 & $1 / 3$ & $2 / 3$ & 1 & 0 \\
$\kappa^{\mathrm{bf}}$ & 0 & 0 & 0 & 0 & 1 \\
\hline
\end{tabular}

where $\tau^{\max }$ and $\tau^{\text {min }}$ are, respectively, the maximum and minimum pheromone values allowed. Hence, $c f=0$ when the algorithm is initialized (or reset), that is, when all pheromone values are set to 0.5 . In contrast, when the algorithm has converged, then $c f=1$. In all other cases, $c f$ has a value within $(0,1)$.

The next step of the algorithm updates the pheromone information by means of the procedure ApplyPheromoneUpdate $\left(c f, b s \_u p d a t e, \mathcal{T}, P^{\mathrm{ib}}, P^{\mathrm{rb}}, P^{\mathrm{bf}}\right)$. In general, three solutions are used for updating the pheromone values. These are the iteration-best solution $P^{\mathrm{ib}}$, the restart-best solution $P^{\mathrm{rb}}$, and the best-so-far solution $P^{\mathrm{bf}}$. The influence of each solution on the pheromone update depends on the state of convergence of the algorithm as measured by the convergence factor $c f$. Hence, each pheromone value $\tau_{i j} \in \mathcal{T}$ is updated as follows:

$$
\tau_{i j}=\tau_{i j}+\rho \cdot\left(\xi_{i j}-\tau_{i j}\right),
$$

with

$$
\xi_{i j}=\kappa^{\mathrm{ib}} \cdot P_{i j}^{\mathrm{ib}}+\kappa^{\mathrm{rb}} \cdot P_{i j}^{\mathrm{rb}}+\kappa^{\mathrm{bf}} \cdot P_{i j}^{\mathrm{bf}},
$$

where $\rho$ is a parameter that determines the learning rate, $P_{i j}^{*}$ is 1 if customer $j$ is visited after customer $i$ in solution $P^{*}$ and 0 otherwise, $\kappa^{\mathrm{ib}}$ is the weight (that is, the influence) of solution $P^{\mathrm{ib}}, \kappa^{\mathrm{rb}}$ is the weight of solution $P^{\mathrm{rb}}, \kappa^{\mathrm{bf}}$ is the weight of solution $P^{\mathrm{bf}}$, and $\kappa^{\mathrm{ib}}+\kappa^{\mathrm{rb}}+\kappa^{\mathrm{bf}}=1$. Equation (8) allows to choose how to schedule the relative influence of the three solutions used for updating the pheromone values. For our application we used a standard update schedule as shown in Table 1 and a value of $\rho=0.1$.

After the pheromone update rule in Eq. (7) is applied, pheromone values that exceed $\tau^{\max }=0.999$ are set back to $\tau^{\max }$ ( similarly for $\tau^{\min }=0.001$ ). This is done in order to avoid a complete convergence of the algorithm, which is a situation that should be avoided. This completes the description of our ACO approach for the TSPTW problem.

\section{Beam-ACO with Stochastic Sampling}

As mentioned before, a Beam-ACO algorithm is obtained from a standard ACO algorithm by the replacement of the independent construction of solutions with a probabilistic beam search procedure. The probabilistic beam search that we 


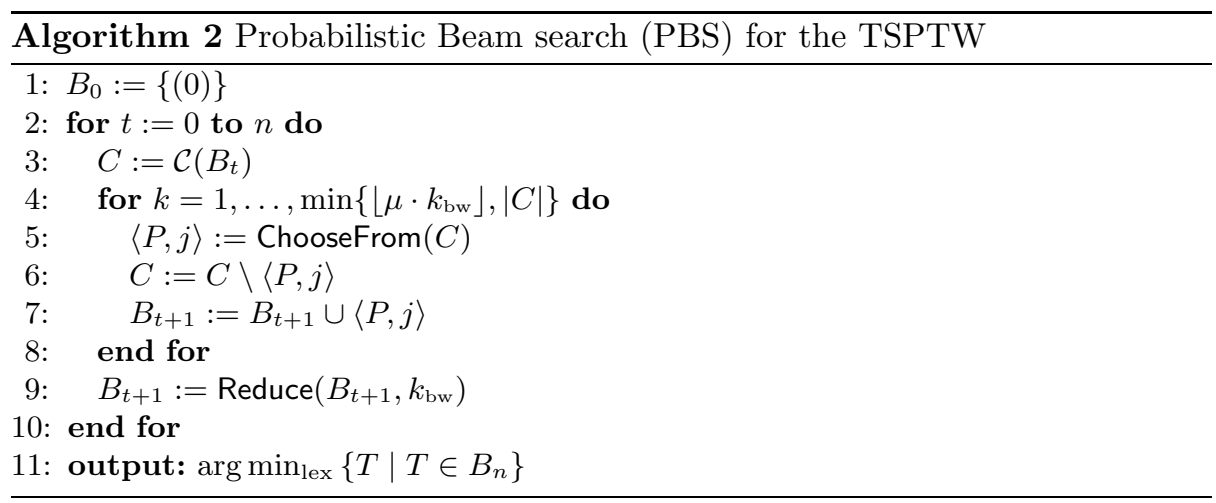

invented for the TSPTW is described in Algorithm 2. The algorithm requires three input parameters: $k_{\mathrm{bw}} \in \mathbb{Z}^{+}$is the so-called beam width, $\mu \in \mathbb{R}^{+} \geq 1$ is a parameter that determines the number of children that can be chosen at each step, and $N^{\mathrm{s}}$ is the number of stochastic samples taken for evaluating a partial solution. Moreover, $B_{t}$ denotes a set of partial tours called the beam. Hereby, index $t$ denotes the current iteration of the beam search. At any time it holds that $\left|B_{t}\right| \leq k_{\mathrm{bw}}$, that is, the beam is smaller or equal than the beam width. A problem-dependent greedy function $\nu()$ is utilized to assign a weight to partial solutions.

At the start of the algorithm the beam only contains one partial tour starting at the depot, that is, $B_{0}:=\{(0)\}$. Let $C:=\mathcal{C}\left(B_{t}\right)$ denote the set of all possible extensions of the partial tours in $B_{t}$. A partial tour $P$ may be extended by adding a customer $j$ not yet visited by that tour. Such a candidate extension of a partial tour is henceforth denoted by $\langle P, j\rangle$. At each iteration, at most $\left\lfloor\mu \cdot k_{\mathrm{bw}}\right\rfloor$ candidate extensions are selected from $C$ by means of the procedure ChooseFrom $(C)$ to form the new beam $B_{t+1}$. At the end of each step, the new beam $B_{t+1}$ is reduced by means of the procedure Reduce in case it contains more than $k_{\mathrm{bw}}$ partial solutions. When the current iteration is equal to the number of customers $(t=n)$, all elements in $B_{n}$ are completed by adding the depot, and finally the best solution is returned.

The procedure ChooseFrom $(C)$ chooses a candidate extension $\langle P, j\rangle$ from $C$, either deterministically or probabilistically according to a parameter $q_{0}$ called determinism rate (see also the description of ACO). More precisely, for each call to ChooseFrom $(C)$, a random number $q$ is generated and if $q \leq q_{0}$ then the decision is taken deterministically by choosing the candidate extension that maximises the product of the pheromone information $\mathcal{T}$ and the greedy function $\nu()$ :

$$
\langle P, j\rangle=\arg \max _{\left\langle P^{\prime}, k\right\rangle \in C} \tau\left(\left\langle P^{\prime}, k\right\rangle\right) \cdot \nu\left(\left\langle P^{\prime}, k\right\rangle\right)^{-1}
$$

where $\tau\left(\left\langle P^{\prime}, k\right\rangle\right)$ corresponds to the pheromone value $\tau_{i k} \in \mathcal{T}$, supposing that $i$ is the last customer visited in tour $P^{\prime}$. 
Otherwise, if $q>q_{0}$, the decision is taken stochastically according to the following probabilities:

$$
\mathbf{p}(\langle P, j\rangle)=\frac{\tau(\langle P, j\rangle) \cdot \nu(\langle P, j\rangle)^{-1}}{\sum_{\left\langle P^{\prime}, k\right\rangle \in C} \tau\left(\left\langle P^{\prime}, k\right\rangle\right) \cdot \nu\left(\left\langle P^{\prime}, k\right\rangle\right)^{-1}}
$$

The greedy function $\nu(\langle P, j\rangle)$ assigns a heuristic value to each candidate extension $\langle P, j\rangle$. In principle, for this purpose we could use the heuristic $\eta$ given by Eq. (4), that is, $\nu(\langle P, j\rangle)=\eta(\langle P, j\rangle)$. As in the case of the pheromone information, the notation $\eta(\langle P, j\rangle)$ refers to the value of $\eta_{i k}$ as defined in Eq. (4), supposing that $i$ was the last customer visited in tour $P$. However, when comparing two extensions $\langle P, j\rangle \in C$ and $\left\langle P^{\prime}, k\right\rangle \in C$, the value of $\eta$ might be misleading in case $P \neq P^{\prime}$. We solved this problem by defining the greedy function $\nu()$ as follows.

Firstly, instead of using the value of $\eta$ directly, we rank the extensions with respect to their value of $\eta$ and use the corresponding ranks for comparison. More specifically, the extension with the highest value of $\eta$ for all candidate extensions of the same tour receives rank 1. Formally, $r(\langle P, j\rangle)=1$ where $\langle P, j\rangle=\arg \max _{k \in \mathcal{N}(P)} \eta(\langle P, k\rangle)$. The extension with the second highest value of $\eta$ receives rank 2, and so on and so forth. Secondly, the value of the greedy function of an extension $\nu(\langle P, j\rangle)$ is calculated as the sum of the ranks that correspond to the sequence of extensions generated during the construction of partial tour $P=\left\{p_{0}, p_{1}, \ldots, p_{|P|}\right\}$. Formally:

$$
\nu(\langle P, j\rangle)=r\left(\left\langle 0, p_{1}\right\rangle\right)+\left(\sum_{i=2}^{|P|} r\left(\left\langle\left(p_{0}, \ldots, p_{i-1}\right), p_{i}\right\rangle\right)\right)+r(\langle P, j\rangle),
$$

where $p_{0}=0$ is the depot, and $p_{i}$ denotes the index of the customer visited in the $i^{\text {th }}$ position of the tour. This definition of $\nu()$ allows us to compare extensions of different partial tours by giving more priority to those extensions maximising $\nu()^{-1}$.

Finally, the application of procedure $\operatorname{Reduce}\left(B_{t}\right)$ removes the worst $\max \left\{\left|B_{t}\right|-\right.$ $\left.k_{\mathrm{bw}}, 0\right\}$ partial solutions from $B_{t}$. As mentioned before, in standard applications of beam search, the worst solutions are determined by applying - in the case of minimization - a lower bound to each partial solution. The solutions removed from $B_{t}$ are then the ones with the greatest lower bound value. However, as the literature for the TSPTW does not offer accurate and at the same time computationally inexpensive lower bounds, we use stochastic sampling for evaluating partial solutions. More specifically, a sample of a partial solution is obtained by using an ant (from the standard ACO algorithm) to complete the tour by iteratively adding unvisited customers following Eq. (2). For each partial solution, a number $N^{\mathrm{s}}$ of complete solutions is sampled. The value of the best of these samples (with respect to Eq. 5) is used for evaluating the corresponding partial solution. Only the $k_{\mathrm{bw}}$ best partial solutions (with respect to their corresponding best samples) are kept in $B_{t}$ and the others are discarded. 
The above procedure defines a probabilistic beam search algorithm, henceforth denoted by $\operatorname{PBS}\left(k_{\mathrm{bw}}, \mu, N^{\mathrm{s}}\right)$. In Beam-ACO, this PBS algorithm replaces the construction loop performed by the ants in Algorithm 1 (lines 5-13). Instead, a single call to PBS generates the iteration-best solution.

\section{Experimental Evaluation}

We implemented $\mathrm{ACO}$ and Beam-ACO in $\mathrm{C}++$. The algorithms were tested on 30 instances provided by Potvin and Bengio [10] and derived from Solomon's RC2 VRPTW instances [11]. These instances are known to contain a mix of randomlyspaced and clustered customers. First, we performed a set of initial experiments in order to find appropriate values for various parameters of ACO and BeamACO. Next, we compared the results obtained by the two algorithms for the 30 instances mentioned above. All experiments were run on a AMD Opteron 8218 processor, with 2.6 GHz CPU and $1 \mathrm{MB}$ of cache size running GNU/Linux 2.6.24.

Comparing different algorithms for the TSPTW is not a trivial task. In the literature it can sometimes be observed that algorithms are compared with respect to their average number of constraint violations and their average cost, where average refers to the average over several runs. However, in general one is not interested in trading a lower tour cost for a higher number of constraint violations. Comparing the two averages mentioned above, it is difficult to assess if such a trade-off has indeed occurred. As an alternative, one might focus on the median tour cost of those runs that achieved the minimum number of constraint violations. Yet, this information does not summarise the typical behaviour of an algorithm, and does therefore not provide a means for a fair comparison. (Imagine, for example, a situation in which one of the algorithms has achieved its minimum number of constraint violations in only one run.)

Instead, we decided for the following mechanism for the comparison of two or more algorithms. More specifically, we calculate a score for each algorithm, ${ }^{1}$ measuring the quality of its solutions relative to the quality of the solutions obtained by the competing approaches. The score is given by the percentage of times that the outcome of one algorithm was better than the outcomes obtained by the alternatives minus the percentage of times that the outcome of the same algorithm was worse than the outcomes obtained by the alternatives. For example, let us compare three different algorithms $X, Y, Z$. Let us assume that each algorithm is applied 5 times to a problem instance. Then, we calculate the score of, for example, algorithm $X$ as follows. First, each of the 5 solutions obtained by $X$ are compared with each of the 10 solutions obtained by $Y$ and $Z$. The comparison is done lexicographically, following the order defined in Eq. (5), by considering first the number of constraint violations $(\Omega)$ and next the tour cost $(F)$. Hence, for each comparison, a tour may be better, equal, or worse than another. We count the number of times that an outcome of $X$ was better minus

\footnotetext{
${ }^{1}$ Here, the term algorithm may refer to ACO or Beam-ACO, or to different configurations of $\mathrm{ACO}$ and Beam-ACO.
} 
the number of times it was worse than the solutions produced by competing algorithms. Finally, we calculate the percentage with respect to the total number of pairwise comparisons, 50 in our example. A positive score indicates that the solutions obtained by $X$ were more often better than the solutions obtained by the alternatives $Y$ and $Z$. A negative score indicates that the alternative algorithms obtained more often better outcomes than $X$. A value close to zero either indicates that the outcomes were most of the times equal or that $X$ obtained as many better outcomes as worse outcomes than the rest.

We applied the ACO algorithm to each instance with various values for the number of ants, that is, $N^{\mathrm{a}}=\{10,20,50,100,200\}$. The rest of the parameters of ACO were set as follows: $\tau^{\max }=0.999, \tau^{\min }=0.001, q_{0}=0.9$, and $\rho=$ 0.1. Each run of ACO was stopped after $15 \mathrm{CPU}$ seconds and we repeated each experiment 25 times with different random seeds. Table 2 gives the scores obtained by ACO for each value of $N^{\mathrm{a}}$. As discussed above, at each row of the table, each entry represents a percentage score obtained by the difference of two values: the number of times that a tour obtained by ACO using the number of ants given in the column heading was better than a tour obtained when using a different number of ants, minus the number of times that the former was worse than the latter. Therefore, larger positive values indicate a higher (relative) quality of the results in comparison with the other values of $N^{\mathrm{a}}$, while negative values indicate a worse quality of the tours obtained. The results in Table 2 show that the best setting of $N^{\mathrm{a}}$ varies depending on the particular instance. Although higher values of $N^{\mathrm{a}}$ lead to a better overall performance, they result in significantly worse results for a few instances, such as rc203.1 and rc204.3. This suggests that instances have important structural differences that are not reflected by their corresponding number of customers $n$.

In a similar manner, Table 3 shows the scores obtained by Beam-ACO when using different settings of $k_{\mathrm{bw}}$ and $N^{\mathrm{s}}$. We decided to study all combinations between $k_{\mathrm{bw}}=\{10,20,30,40,50\}$ and $N^{\mathrm{s}}=\{1,5,10,20\}$. The remaining BeamACO parameters were set in the same way as for ACO, except for the beamsearch parameter $\mu=1.5$. We applied each configuration of Beam-ACO 25 times for $15 \mathrm{CPU}$ seconds to each test instance. Each table cell in Table 3 gives the score obtained by Beam-ACO using the $k_{\mathrm{bw}}$ and $N^{\mathrm{s}}$ settings given by the column with respect to the results obtained by all the other configurations of Beam-ACO in the same row. Again, the best configuration per instance is indicated in boldface. Note that, for example, for instance rc201.1 all configurations obtained the optimal solution in all runs. In such cases, we indicate in boldface the configuration that required less median CPU time.

The best settings of $k_{\mathrm{bw}}$ and $N^{\mathrm{s}}$ depend strongly on the particular instance, as shown in Table 3 . In most cases, a small beam-width $\left(k_{\mathrm{bw}}=10\right)$ and number of samples $N^{\mathrm{s}} \in\{1,5\}$ obtained the best solutions. However, in certain cases, these settings produced notably worse results and higher values of $k_{\mathrm{bw}}$ and $N^{\mathrm{s}}$ are required, as for example when instances rc204.3 and rc205.1 are concerned.

Finally, a comparison between ACO and Beam-ACO is presented in Table 4. In this comparison, we used for each instance and for each algorithm the con- 
figuration that obtained the highest scores in Tables 2 and 3, that is, the ones marked in boldface. For both ACO and Beam-ACO, Table 4 gives the median $(\tilde{\Omega})$, standard deviation (sd), and minimum $\left(\Omega_{\mathrm{min}}\right)$ number of constraint violations obtained in 25 runs, then the number of runs $([\#])$ that obtained that minimum number of constraint violations. For those runs that obtained $\Omega_{\min }$ constraint violations, $\tilde{F}$ and sd give the median and standard deviation tour cost and $\tilde{T}_{\text {cpu }}$ and sd are the median and standard deviation CPU time (in seconds). Finally, we calculate the score (column "Score") of Beam-ACO with respect to $\mathrm{ACO}$ as described earlier. In other words, we compare each of the 25 solutions obtained by Beam-ACO with each of the 25 solutions generated by ACO, for each instance. Next, for the resulting 625 pairwise comparisons, we count the percentage of how many times Beam-ACO was better minus how many times it was worse than ACO. Hence, a positive score indicates that the solutions generated by Beam-ACO were more frequently better than those obtained by ACO.

Table 4 shows, first, that Beam-ACO obtains a positive score in 20 out of 30 cases. Of the remaining 10 instances, 5 appear to be excessively easy for both algorithms, since both reach an optimal solution in all runs. Only in 5 instances ACO obtains a better score than Beam-ACO. Nevertheless, comparing the median tour costs $(\tilde{F})$ reveals that the advantage of ACO is quite small. In summary, we can conclude that Beam-ACO based on stochastic sampling provides an evident advantage over the non-hybrid ACO algorithm when applied to the 30 test instances used in this study. This becomes also clear when studying the columns with heading [\#]: Beam-ACO is generally more robust in finding solutions with a low number of constraint violations.

\section{Conclusions}

In this paper, we have proposed a Beam-ACO approach for the TSPTW. BeamACO is a hybrid between ant colony optimization and beam search that relies heavily on bounding information that is accurate and computationally inexpensive. We studied a new version of Beam-ACO in which the bounding information is replaced by stochastic sampling. We performed experiments on a set of standard benchmark instances for the TSPTW, comparing a pure ACO algorithm with Beam-ACO based on stochastic sampling. The results showed that BeamACO obtains generally better results in most instances. In a few instances, ACO achieved slightly better results than Beam-ACO. In those instances, the heuristic information is probably quite deceptive. Nonetheless, the overall positive performance of Beam-ACO based on stochastic sampling in comparison to ACO shows that Beam-ACO can be useful even when no accurate and computationally inexpensive bounding information is available.

In the future we plan to improve the performance of our Beam-ACO approach further, for example, by the inclusion of local search and by the study of different types of heuristic information. 


\section{Acknowledgements}

We wish to thank Professor Ohlmann for making widely available the benchmark instances used in his work.

\section{References}

1. Dorigo, M., Stützle, T.: Ant Colony Optimization. MIT Press (2004)

2. Blum, C.: Beam-ACO-hybridizing ant colony optimization with beam search: an application to open shop scheduling. Computers \& Operations Research 32 (2005) $1565-1591$

3. Blum, C.: Beam-ACO for simple assembly line balancing. INFORMS Journal on Computing (2008) In press.

4. Ow, P.S., Morton, T.E.: Filtered beam search in scheduling. International Journal of Production Research 26 (1988) 297-307

5. Juillé, H., Pollack, J.B.: A sampling-based heuristic for tree search applied to grammar induction. In: Proceedings of AAAI 1998 - Fifteenth National Conference on Artificial Intelligence, MIT press (1998) 776-783

6. Ruml, W.: Incomplete tree search using adaptive probing. In: Proceedings of IJCAI 2001 - Seventeenth International Joint Conference on Artificial Intelligence, IEEE press (2001) 235-241

7. Savelsbergh, M.W.P.: Local search in routing problems with time windows. Annals of Operations Research 4(1) (December 1985) 285-305

8. Ohlmann, J.W., Thomas, B.W.: A compressed-annealing heuristic for the traveling salesman problem with time windows. INFORMS Journal on Computing 19(1) (2007) 80-90

9. Blum, C., Dorigo, M.: The hyper-cube framework for ant colony optimization. IEEE Transactions on Systems, Man, and Cybernetics - Part B 34(2) (2004) 11611172

10. Potvin, J.Y., Bengio, S.: The vehicle routing problem with time windows part II: Genetic search. INFORMS Journal on Computing 8 (1996) 165-172

11. Solomon, M.M.: Algorithms for the vehicle routing and scheduling problems with time windows. Operations Research 35 (1987) 254-265 
Table 2. Relative scores obtained by ACO with different settings of $N^{\mathrm{a}}$.

\begin{tabular}{|c|c|c|c|c|c|c|}
\hline \multicolumn{2}{|c|}{ Instance } & \multicolumn{5}{|c|}{$N^{\mathrm{a}}$} \\
\hline Problem & $n$ & 10 & 20 & 50 & 100 & 200 \\
\hline rc201.1 & 19 & 0.0 & 0.0 & 0.0 & 0.0 & 0.0 \\
\hline rc201.2 & 25 & 11.3 & 27.8 & -14.1 & 0.9 & -25.9 \\
\hline rc201.3 & 31 & -45.9 & -7.3 & 19.1 & 23.6 & 10.6 \\
\hline rc201.4 & 25 & -50.2 & -48.6 & -0.6 & 45.3 & 54.0 \\
\hline rc202.1 & 32 & -37.1 & -37.3 & 15.4 & 26.0 & 32.9 \\
\hline rc202.2 & 13 & 12.9 & -3.2 & -19.3 & -5.5 & 15.2 \\
\hline rc202.3 & 28 & -27.5 & -19.3 & 13.1 & 22.3 & 11.4 \\
\hline rc202.4 & 27 & -30.4 & -29.0 & 0.8 & 38.5 & 20.0 \\
\hline rc203.1 & 18 & -0.8 & 50.2 & 37.4 & -3.7 & -83.0 \\
\hline rc203.2 & 32 & -27.2 & -26.1 & 17.0 & 12.7 & 23.6 \\
\hline rc203.3 & 36 & -20.7 & -20.0 & -4.0 & 14.0 & 30.7 \\
\hline rc203.4 & 14 & -74.2 & 9.0 & 1.0 & 34.4 & 29.8 \\
\hline rc204.1 & 44 & -21.0 & -10.2 & 7.0 & 18.6 & 5.8 \\
\hline rc204.2 & 32 & -12.4 & 1.4 & 19.6 & -1.9 & -6.8 \\
\hline rc204.3 & 33 & 65.2 & 32.4 & -21.2 & -40.9 & -35.6 \\
\hline rc205.1 & 13 & -3.0 & -3.0 & 2.0 & 2.0 & 2.0 \\
\hline rc205.2 & 26 & -41.6 & -52.2 & 16.4 & 27.0 & 50.4 \\
\hline rc205.3 & 34 & 18.0 & -2.2 & -53.2 & 0.0 & 37.4 \\
\hline rc205.4 & 27 & -38.2 & -17.0 & 34.2 & 4.8 & 16.1 \\
\hline rc206.1 & 3 & 0.0 & 0.0 & 0.0 & 0.0 & 0.0 \\
\hline rc206.2 & 36 & 6.8 & -8.5 & -24.0 & 5.8 & 20.0 \\
\hline rc206.3 & 24 & -56.9 & -20.8 & 19.8 & 37.8 & 20.0 \\
\hline rc206.4 & 37 & -8.4 & -35.6 & 10.7 & 9.3 & 24.0 \\
\hline rc207.1 & 33 & -26.2 & -13.4 & 22.3 & 0.0 & 17.3 \\
\hline rc207.2 & 30 & 5.2 & 5.4 & -27.5 & 6.7 & 10.2 \\
\hline rc207.3 & 32 & -46.8 & 14.0 & 4.9 & 17.6 & 10.2 \\
\hline rc207.4 & 5 & 0.0 & 0.0 & 0.0 & 0.0 & 0.0 \\
\hline rc208.1 & 37 & -26.3 & 4.6 & 2.0 & -1.1 & 20.9 \\
\hline rc208.2 & 28 & -66.7 & -13.0 & 37.0 & 15.3 & 27.4 \\
\hline rc208.3 & 35 & -26.4 & -10.8 & 5.0 & 2.3 & 29.9 \\
\hline
\end{tabular}


Table 3. Relative scores obtained by Beam-ACO for different values of $k_{\mathrm{bw}}$ and $N^{\mathrm{s}}$.

\begin{tabular}{|c|c|c|c|c|c|c|c|c|c|c|c|c|c|c|c|c|c|c|c|c|c|}
\hline \multicolumn{2}{|c|}{ Instance } & \multicolumn{4}{|c|}{$k_{\mathrm{bw}}=10$} & \multicolumn{4}{|c|}{$k_{\mathrm{bw}}=20$} & \multicolumn{4}{|c|}{$k_{\mathrm{bw}}=30$} & \multicolumn{4}{|c|}{$k_{\mathrm{bw}}=40$} & \multicolumn{4}{|c|}{$k_{\mathrm{bw}}=50$} \\
\hline Problem & $n$ & $=1$ & 5 & 10 & 20 & $=1$ & 5 & 10 & 20 & $=1$ & 5 & 10 & 20 & $\mathrm{~s}=1$ & 5 & 10 & 20 & $\mathrm{~s}=1$ & 5 & 10 & 20 \\
\hline rc201.1 & 19 & 0.0 & 0.0 & 0.0 & 0.0 & 0.0 & 0.0 & 0.0 & 0.0 & 0.0 & 0.0 & 0.0 & 0.0 & 0.0 & 0.0 & 0.0 & 0.0 & 0.0 & 0.0 & 0.0 & 0.0 \\
\hline & & & 27.4 & 18.2 & & & & -1.3 & -9.2 & & -47.2 & & -31 & & -32.1 & -23.2 & & & -4.0 & & \\
\hline & 31 & & 15.1 & 1.1 & 19.8 & & 14.4 & 8.3 & -8.0 & & 15.5 & 7.0 & & & 13.6 & 13.1 & & & 22.5 & 4.0 & 027 \\
\hline & 25 & & 14.8 & 26.1 & 26.1 & & 14.8 & 22.3 & 26.1 & -29.5 & 3.6 & 18.6 & 14.8 & 2.9 & -1.3 & 14.8 & 26.1 & D1.5 & -5.0 & 7.3 & $\begin{array}{ll}3 & -5\end{array}$ \\
\hline rc202.1 & 32 & & 20.2 & 43.1 & 27.7 & -26.3 & 9.6 & 30.0 & .5 & -25.6 & 3.6 & 4.3 & 18.4 & 33.6 & -14.0 & -12.9 & -10.8 & 33.3 & -6.0 & 10.4 & $\begin{array}{ll}4 & 22.5\end{array}$ \\
\hline & & & & & & & & & & & & & & & & & & & & & \\
\hline & & & 43.4 & 19.2 & 40.6 & .2 & 21.4 & 22.6 & -5.0 & -17.2 & 4.3 & -4.2 & -5.7 & & 8.1 & -4.8 & & -41.8 & -14.9 & -15.7 & $7-32$ \\
\hline 4 & 27 & & -47.5 & -38.6 & -45.8 & -0.2 & 35.1 & 27.9 & 3.1 & -2.3 & 1.5 & 14.6 & 2.2 & 19.2 & 2.5 & 12.5 & & 21.8 & 51.1 & 5.3 & 6.3 \\
\hline $\mathrm{rc} 203.1$ & & .7 & 34.7 & 34.7 & 28.7 & 34.7 & 4.8 & -22.9 & -35.9 & 34.7 & 28.7 & -28.9 & -60.9 & 34.7 & 5.9 & -40.6 & -45.5 & 31.1 & 18.0 & 257 & -65.3 \\
\hline & & & 1.1 & & & & 33.3 & & & & & 7.2 & & & & & & & & & \\
\hline & & & -17.0 & & 0. & & & & & & & & & & & & & & & & \\
\hline & 14 & & 2.0 & -11.6 & 20.4 & 3.1 & 19.8 & -0.3 & 2.9 & 32.5 & 15.5 & -3.3 & -10.7 & 51.5 & -7.8 & -29.3 & -31.4 & .3 & -5 & 0.1 & -29.6 \\
\hline & & & 0 & & 42.6 & & 2.7 & 12.9 & 15 & -33.4 & 10.6 & -3.9 & 10. & -30.2 & -1.7 & -14.6 & -25.8 & 2.1 & -8.2 & 36 & -32 \\
\hline & & & & & & & 23.4 & & 4.6 & & 6.3 & -33.2 & & 1. & -2.0 & & & & & & \\
\hline rc204.3 & 33 & -32 & -48.2 & -15.1 & 13.2 & -13.1 & -34.8 & -50.7 & 27.8 & -45.7 & -8.1 & 27.9 & 54.0 & -41.1 & 31.7 & 45.3 & 42.5 & -34.2 & 27.7 & 22.9 & 30.3 \\
\hline & & & 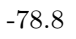 & -5 & -60 & & 4.8 & & -12 & & & .4 & & & 35.4 & 35.4 & & & 31.9 & 35.4 & 17. \\
\hline & & & & & & & & & & & & & & & & & & & .0 & & 217 \\
\hline & & & & & & & & -2 & & & 17.3 & -10.4 & & & -22.5 & -9.5 & & & & & \\
\hline & 27 & & 77.7 & 47.2 & 25.1 & 52.6 & 12.5 & 6.9 & -25.8 & 2.3 & -9.6 & 4.6 & -56 & -13 & -7.8 & -13.1 & -39.7 & -1 & -33 & & $3-5$ \\
\hline & 3 & & & & & & & & & 0 & 0.0 & 0.0 & & 0 & 0.0 & م0 & & 00 & 0 & 0 & 00 \\
\hline & & & .6 & & & & & & & & & & & & & & & & & & \\
\hline & & & & & & & & & & & & & & & & & & & & & \\
\hline & 37 & & 40.7 & 12.9 & -47.9 & -6.6 & 38.8 & & -40.5 & 2.3 & 17.7 & -34.2 & -26 & -14 & 37.4 & -6 & & .5 & 30.5 & 22.1 & 24.1 \\
\hline & & & & & & & & & -38.0 & 5.2 & & -27.5 & -34 & & -15.0 & -19.2 & & 1.8 & -32.0 & -29.7 & -30.6 \\
\hline & & & & & 53. & & & & & & & & & & & & & & & & \\
\hline & 32 & & 32.5 & 66.0 & & & & -3.8 & 35 & & 19 & -4.8 & & & -26.9 & 1.3 & -42 & & -36.8 & & \\
\hline & 5 & 0.0 & 0.0 & 0.0 & 0.0 & 0.0 & 0.0 & 0.0 & 0.0 & 0.0 & 0.0 & 0.0 & 0.0 & 0.0 & 0.0 & 0.0 & 0.0 & 0.0 & 0.0 & 0.0 & 0.0 \\
\hline & & & & & & & & & & & & 14.7 & & & & & & & & & \\
\hline & 2 & & & & 41 & & 17.5 & -9 & & & & & & & -11.4 & -11.8 & -44 & & -22.0 & -28.5 & -22 \\
\hline rc208.3 & 35 & -40.1 & 44.4 & 31.8 & 37.2 & -52.2 & 16.7 & 24.0 & 11.2 & -48.2 & 15.6 & 13.4 & 8.7 & -79.2 & 9.1 & 30.2 & 15.3 & -65.1 & 15.4 & 12.4 & -0.6 \\
\hline
\end{tabular}


Table 4. Comparison of the best results of ACO and Beam-ACO for each instance.

\begin{tabular}{|c|c|c|c|c|c|c|c|c|c|c|c|c|c|c|c|c|c|c|}
\hline \multicolumn{2}{|c|}{ Instance } & \multicolumn{8}{|c|}{ Beam-ACO } & \multicolumn{8}{|c|}{$\mathrm{ACO}$} & \multirow[t]{2}{*}{ Score } \\
\hline Problem & $n$ & $\tilde{\Omega}$ & $\mathrm{sd}$ & $\Omega_{\min }$ & [\#] & $\tilde{F}$ & $\mathrm{sd}$ & $\tilde{T}_{\mathrm{cpu}}$ & $\mathrm{sd}$ & $\tilde{\Omega}$ & $\mathrm{sd}$ & $\Omega_{\min }$ & {$[\#]$} & $\tilde{F}$ & $\mathrm{sd}$ & $\tilde{T}_{\text {cpu }}$ & sd & \\
\hline rc201.1 & 19 & 0 & 0.00 & 0 & 25 & 444.54 & 0.00 & 0.01 & 0.00 & 0 & 0.00 & 0 & 25 & 444.54 & 0.00 & 0.27 & 0.38 & 0 \\
\hline $\mathrm{rc} 201.2$ & 25 & 0 & 0.00 & 0 & 25 & 712.91 & 2.48 & 8.28 & 3.36 & 1 & 0.58 & 0 & 12 & 723.14 & 7.92 & 10.18 & 2.59 & 93.8 \\
\hline $\mathrm{rc} 201.3$ & 31 & 0 & 0.00 & 0 & 25 & 797.25 & 2.36 & 5.40 & 4.37 & 0 & 0.41 & 0 & 20 & 806.26 & 3.57 & 10.71 & 3.03 & 88.5 \\
\hline rc201.4 & 25 & 0 & 0.00 & 0 & 25 & 793.64 & 0.00 & 0.75 & 1.68 & 0 & 0.00 & 0 & 25 & 793.64 & 0.00 & 3.74 & 3.17 & 0 \\
\hline $\mathrm{rc} 202.1$ & 32 & 0 & 0.00 & 0 & 25 & 782.77 & 4.39 & 11.44 & 2.82 & 0 & 0.51 & 0 & 13 & 788.47 & 11.45 & 13.27 & 0.93 & 78.2 \\
\hline $\mathrm{rc} 202.2$ & 13 & 0 & 0.00 & 0 & 25 & 304.14 & 0.00 & 4.27 & 3.54 & 0 & 0.00 & 0 & 25 & 304.14 & 0.25 & 6.45 & 3.38 & 12 \\
\hline $\mathrm{rc} 202.3$ & 28 & 0 & 0.00 & 0 & 25 & 852.35 & 11.03 & 8.07 & 3.91 & 0 & 0.33 & 0 & 22 & 846.70 & 12.78 & 7.53 & 4.21 & 7.8 \\
\hline $\mathrm{rc} 202.4$ & 27 & 0 & 0.59 & 0 & 14 & 854.74 & 16.02 & 11.32 & 3.31 & 1 & 0.20 & 0 & 1 & 860.75 & - & 4.89 & - & 31.5 \\
\hline $\mathrm{rc} 203.1$ & 18 & 0 & 0.00 & 0 & 25 & 453.48 & 0.00 & 3.02 & 2.39 & 0 & 0.00 & 0 & 25 & 453.48 & 0.57 & 6.32 & 4.34 & 20 \\
\hline $\mathrm{rc} 203.2$ & 32 & 0 & 0.33 & 0 & 22 & 844.13 & 17.82 & 11.52 & 2.72 & 0 & 0.41 & 0 & 20 & 837.40 & 29.36 & 13.67 & 3.70 & 3.7 \\
\hline rc203.3 & 36 & 1 & 0.20 & 1 & 24 & 857.43 & 7.98 & 10.49 & 2.72 & 1 & 0.55 & 0 & 1 & 852.86 & - & 11.13 & - & 78.6 \\
\hline rc203.4 & 14 & 0 & 0.00 & 0 & 25 & 321.35 & 2.6 & 5.09 & 4.50 & 0 & 0.00 & 0 & 25 & 319.45 & 2.1 & 4.58 & 3.91 & -45.9 \\
\hline rc204.1 & 44 & 1 & 0.99 & 0 & 7 & 940.17 & 9.83 & 13.81 & 2.03 & 1 & 0.84 & 0 & 7 & 938.20 & 0.95 & 13.02 & 2.35 & -3.7 \\
\hline $\mathrm{rc} 204.2$ & 32 & 0 & 0.00 & 0 & 25 & 684.13 & 8.65 & 11.85 & 2.77 & 0 & 0.00 & 0 & 25 & 692.17 & 9.44 & 10.78 & 3.25 & 46.4 \\
\hline $\mathrm{rc} 204.3$ & 33 & 0 & 0.00 & 0 & 25 & 461.93 & 2.10 & 5.15 & 3.82 & 0 & 0.00 & 0 & 25 & 463.04 & 3.55 & 6.03 & 3.57 & 53.0 \\
\hline $\mathrm{rc} 205.1$ & 13 & 0 & 0.00 & 0 & 25 & 343.21 & 0.00 & 1.82 & 3.23 & 0 & 0.00 & 0 & 25 & 343.21 & 0.00 & 0.84 & 0.79 & 0 \\
\hline $\mathrm{rc} 205.2$ & 26 & 0 & 0.00 & 0 & 25 & 755.93 & 4.33 & 10.22 & 3.68 & 0 & 0.56 & 0 & 18 & 768.78 & 9.58 & 8.33 & 4.05 & 72.6 \\
\hline $\mathrm{rc} 205.3$ & 34 & 2 & 0.00 & 2 & 25 & 816.25 & 2.75 & 8.38 & 4.03 & 2 & 0.00 & 2 & 25 & 816.55 & 3.98 & 7.31 & 4.50 & 23.4 \\
\hline rc205.4 & 27 & 1 & 0.51 & 0 & 12 & 762.41 & 4.72 & 10.73 & 2.29 & 1 & 0.44 & 0 & 1 & 764.72 & - & 9.01 & . & 50.6 \\
\hline rc206.1 & 3 & 0 & 0.00 & 0 & 25 & 117.85 & 0.00 & 0.00 & 0.00 & 0 & 0.00 & 0 & 25 & 117.85 & 0.00 & 0.00 & 0.00 & 0 \\
\hline $\mathrm{rc} 206.2$ & 36 & 0 & 0.00 & 0 & 25 & 830.95 & 1.46 & 7.90 & 3.25 & 0 & 0.00 & 0 & 25 & 845.81 & 1.28 & 7.45 & 4.13 & 100 \\
\hline $\mathrm{rc} 206.3$ & 24 & 0 & 0.00 & 0 & 25 & 575.55 & 0.70 & 3.18 & 3.03 & 0 & 0.00 & 0 & 25 & 574.42 & 2.62 & 4.33 & 3.60 & -52 \\
\hline rc206.4 & 37 & 0 & 0.00 & 0 & 25 & 851.02 & 8.56 & 9.28 & 2.61 & 0 & 0.00 & 0 & 25 & 890.81 & 15.92 & 11.48 & 2.53 & 94.9 \\
\hline rc207.1 & 33 & 0 & 0.00 & 0 & 25 & 757.30 & 12.66 & 10.84 & 3.57 & 0 & 0.20 & 0 & 24 & 772.93 & 16.41 & 12.42 & 2.96 & 55.5 \\
\hline $\mathrm{rc} 207.2$ & 30 & 1 & 0.00 & 1 & 25 & 683.99 & 20.17 & 10.26 & 4.48 & 1 & 0.00 & 1 & 25 & 682.35 & 34.29 & 12.45 & 2.80 & 2.7 \\
\hline $\mathrm{rc} 207.3$ & 32 & 0 & 0.00 & 0 & 25 & 730.04 & 10.02 & 11.33 & 2.21 & 0 & 0.00 & 0 & 25 & 722.58 & 10.13 & 12.40 & 3.09 & -35.4 \\
\hline rc207.4 & 5 & 0 & 0.00 & 0 & 25 & 119.64 & 0.00 & 0.00 & 0.00 & 0 & 0.00 & 0 & 25 & 119.64 & 0.00 & 0.00 & 0.00 & 0 \\
\hline rc208.1 & 37 & 0 & 0.00 & 0 & 25 & 842.04 & 12.79 & 10.90 & 3.65 & 0 & 0.00 & 0 & 25 & 867.05 & 22.55 & 11.55 & 3.20 & 52.3 \\
\hline $\mathrm{rc} 208.2$ & 28 & 0 & 0.00 & 0 & 25 & 550.80 & 7.03 & 12.41 & 2.66 & 0 & 0.00 & 0 & 25 & 542.51 & 8.10 & 11.01 & 2.88 & -32.2 \\
\hline $\mathrm{rc} 208.3$ & 35 & 0 & 0.00 & 0 & 25 & 664.42 & 5.27 & 13.37 & 2.47 & 0 & 0.00 & 0 & 25 & 673.92 & 8.88 & 9.92 & 3.93 & 63.2 \\
\hline
\end{tabular}

